\title{
Ultraviolet stability and contamination analysis of Spectralon diffuse reflectance material
}

\author{
Albert E. Stiegman, MEMBER SPIE \\ Carol J. Bruegge, MEMBER SPIE \\ Jet Propulsion Laboratory \\ California Institute of Technology \\ 4800 Oak Grove Drive \\ Pasadena, California 91109
}

Arthur W. Springsteen, MEMBER SPIE Labsphere Incorporated

P.O. Box 70

North Sutton, New Hampshire 03264

\begin{abstract}
A detailed chemical analysis was carried out on Spectralon, a highly Lambertian, diffuse reflectance material. Results of this investigation unambiguously identified the presence of an organic (hydrocarbon) impurity intrinsic to the commercial material. This impurity could be removed by a vacuum bake-out procedure and was identified as the cause of optical changes (degradation) that occur in the material when exposed to UV light. It was found that when this impurity was removed, the Spectralon material was photochemically stable and maintained its reflectance properties even after extensive solar UV exposure.
\end{abstract}

Subject terms: Spectralon; polytetrafluoroethylene; diffuse reflectance standards; calibration; flight qualification; space exposure environmental testing; Multiangle Imaging SpectroRadiometer (MISR); Earth Observing System.

Optical Engineering 32(4), 799-804 (April 1993).

\section{Introduction}

Spectralon ${ }^{\mathrm{TM}}$ is a diffuse reflectance material that is both highly Lambertian and highly reflective $(>0.94$ from 250 to $2500 \mathrm{~nm}$ ). Spectralon, which is the product of Labsphere, is composed of pure polytetrafluoroethylene (PTFE or Teflon) polymer resin that is compressed into a hard porous white material in a proprietary procedure. The excellent reflectance properties of Spectralon make it an ideal material for use in space as a calibration standard for remote sensing applications. However, the ability of this material to perform in the space environment is contingent on, among other things, its ability to resist environmental effects such as UV radiation, high-energy protons, electrons, and hyperthermal atomic oxygen. This report contains an investigation of the effects of UV radiation on the commercial Spectralon reflectance material with an analysis of the contribution of impurities to its observed behavior.

\section{Effect of UV Radiation on Spectralon}

Tests of the UV stability of the reflectance characteristics of Spectralon were carried out at the Thermophysics Facility of TRW under a contract from the Jet Propulsion Laboratory (JPL). ${ }^{1}$ The samples, maintained under a vacuum, were irradiated with a calibrated 5-kW short-arc xenon lamp for periods of 10,100 and 1000 effective UV solar hours

Paper 22082 received Aug. 26, 1992; accepted for publication Oct. 24, 1992 (C) 1993 Society of Photo-Optical Instrumentation Engineers. $0091-3286 / 93 / \$ 2.00$
(EUVSH). The hemispheric spectral reflectance was monitored in situ as a function of irradiation time with a Beckman DK spectrophotometer. The results of this UV exposure for the Spectralon samples is shown in Fig. 1.

A strong decrease in the reflectivity of the Spectralon samples in the visible to near-UV region of the spectrum $(\lambda<0.5 \mu \mathrm{m})$ is observed during irradiation. The total solar absorbance increases from 0.041 in the unexposed sample to 0.206 in the sample that has experienced 1000 EUVSH. This clearly suggests that the material is undergoing a dramatic photochemical change that results in it becoming highly absorbent. In fact, the exposed Spectralon sample appears visibly yellow to the eye. The observation of this UV instability suggests that Spectralon may be unsuitable in space applications. This, however, is contrary to past experience with the use of fluorocarbon (Teflon) based materials in space where they have been successfully used for applications such as thermal control blankets with little change in their physical or optical properties even after extensive UV exposure. This suggests that the photodegradation may be due to impurities in the Spectralon and not to an inherent degradation of the Teflon itself.

\subsection{Surface Analysis of UV Exposed Sample}

The surface of the Spectralon sample that received 1000 EUVSH and was visibly yellow was analyzed by electron spectroscopy for chemical analysis (ESCA) techniques. This technique gives an analysis of the elemental composition of the surface of the material. The surface composition of unexposed and UV-exposed Spectralon are given in Table 1. 


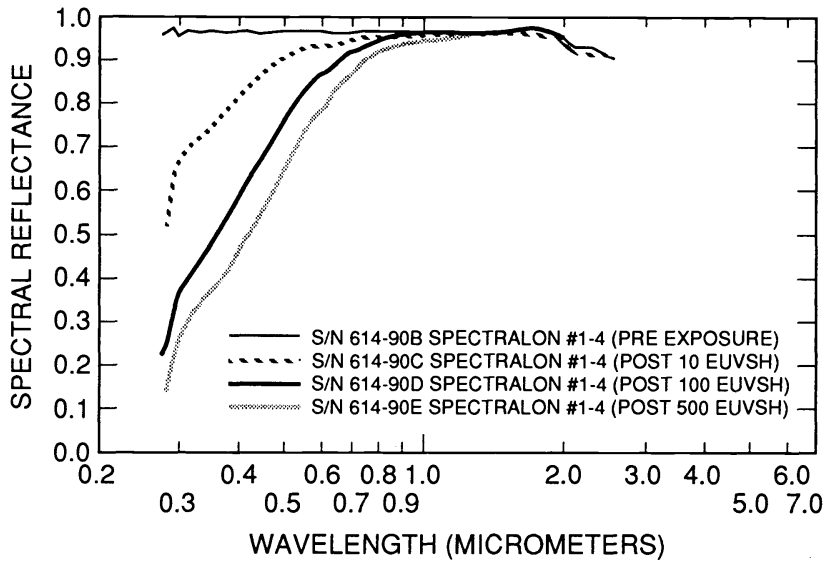

Fig. 1 Total hemispheric reflectance of contaminated Spectralon exposed to solar UV radiation.

The existence of a contaminant can be deduced from the elemental profile by the increase in the percent carbon seen in the irradiated sample. The decrease in the carbon-tofluorine ratio from close to the expected value of 0.5 for pure PTFE to 0.8 is consistent with the existence of one to two monolayers of hydrocarbon on the surface. The presence of oxygen in the irradiated sample suggests that some degree of oxidation takes place at the surface either during photolysis from trace oxygen in the apparatus or from oxidation of photogenerated reactive sites on exposure of the sample to air. One further point can be made from the ESCA data. The fact that the unexposed sample shows no contaminant at the surface does not necessarily mean that it is devoid of contamination, but that the contaminant may be contained within the porous structure of the material where it is not detectable by ESCA. A likely mechanism for the deposition of the yellow organic layer on the irradiated sample is that the impurity diffuses out of the interior of the sample under vacuum, photochemically breaks down as it reaches the surface, and deposits a contamination layer. A mechanism such as this implies that the impurity is volatile and may be detected and possibly removed under vacuum.

\subsection{Mass Spectral Analysis}

Volatile contaminants in the Spectralon sample were analyzed by mass spectroscopy using a procedure devised at JPL specifically for analyzing this material. The Spectralon samples were placed in a vacuum container connected to a mass spectrometer. The container was evacuated by rough pumping ( $10^{-3}$ Torr) for a period of about $30 \mathrm{~min}$, and was then evacuated to high vacuum $\left(10^{-6}\right.$ Torr $)$ by ion pumping. Once a high vacuum was obtained $(\sim 1 \mathrm{~min})$ the sample was sealed off and allowed to outgas for $5 \mathrm{~min}$ at an ambient temperature $\left(22^{\circ} \mathrm{C}\right)$. The volatile effluents were then sampled by mass spectroscopy using a full scan from 4 to 300 amu. Two different Spectralon samples made from two different PTFE resins were analyzed; the results were identical for both. The mass spectrum of the volatile effluents evolved at room temperature is shown in Fig. 2.

The mass spectrum shows predominant mass peaks at 14 (N), 18 (water), $28\left(\mathrm{~N}_{2}, \mathrm{CO}\right.$, or $\left.\mathrm{C}_{2} \mathrm{H}_{4}\right), 32\left(\mathrm{O}_{2}\right)$, which are
Table 1 Surface composition of the Spectralon diffuse reflectance material.

\begin{tabular}{|l|l|l|l|l|}
\hline \multicolumn{2}{|c|}{} & \multicolumn{2}{|c|}{$\begin{array}{l}\text { Control } \\
\text { Sample }\end{array}$} & \multicolumn{2}{c|}{$\begin{array}{c}\text { Exposed } \\
\text { Sample }\end{array}$} \\
\hline Element & Energy & Atom\% & \multicolumn{1}{|c|}{ Energy } & Atom\% \\
\hline \hline C & 290.7 & 31.08 & 284.6 & 43.50 \\
\hline F & 688.4 & 68.91 & 688.9 & 51.58 \\
\hline O & - & - & 532.9 & 4.92 \\
\hline
\end{tabular}

characteristic of air that is still outgassing from the sample. Peaks at 49 and 84 in the spectrum are from residual $\mathrm{CH}_{2} \mathrm{Cl}_{2}$ used to clean the apparatus prior to the experiment. While other peaks appear to be present at low levels, a positive identification of any contaminants is not possible. The temperature of the sample was raised to $50^{\circ} \mathrm{C}$ and allowed to outgas for $1 \mathrm{~h}$ at which point the mass spectrum was recorded. The mass spectrum still primarily shows air outgassing (though in much lower concentrations) with a more complex fragmentation pattern beginning to emerge at higher masses.

By ultimately increasing the temperature to $95^{\circ} \mathrm{C}$ and allowing the sample to outgas for $1.5 \mathrm{~h}$, the complex fragmentation pattern becomes quite visible (Fig. 3).

The large peak at mass 18 in Fig. 3 is the result of residual water, while mass 28 can be $\mathrm{N}_{2}, \mathrm{CO}$ or $\mathrm{C}_{2} \mathrm{H}_{4}$ from the fragmentation of an organic molecule. A detailed analysis of the higher masses confirms the presence of an organic effluent by the characteristic hydrocarbon fragmentation pattern occurring at masses $43\left(\mathrm{C}_{3} \mathrm{H}_{7}\right), 57\left(\mathrm{C}_{4} \mathrm{H}_{9}\right), 71\left(\mathrm{C}_{5} \mathrm{H}_{11}\right)$, and $85\left(\mathrm{C}_{6} \mathrm{H}_{13}\right)$. While the mass spectral analysis confirms the presence of an organic contaminant, it does not permit its exact chemical identification. Furthermore, it is also possible that more than one organic impurity exists contributing to the observed spectrum. Finally, in an attempt to remove the impurity altogether, the sample was ion-pumped overnight at $90^{\circ} \mathrm{C}$ and then closed off and allowed to outgas for $2 \mathrm{~h}$.

The resulting spectrum (Fig. 4) shows that the volatile impurity is almost completely gone with only the ubiquitous mass 28 remaining as a background. Clearly, by application of a vacuum bake-out procedure with the times and temperatures defined by this experiment $\left(90^{\circ} \mathrm{C}, 24 \mathrm{~h}, 10^{-6}\right.$ Torr) the volatile contaminant can be almost completely removed from the material.

\subsection{Extraction of the Spectralon Impurity}

Samples of Spectralon were soxhlet extracted with a freon/ ethanol solution $(9: 1)$ for a period of $24 \mathrm{~h}$. The extraction was carried out in a grease-free glass soxhlet extractor with ultrahigh purity solvents. Removal of the solvent after extraction afforded an oily residue. In an attempt to identify the exact chemical component(s) that composed the impurity, several additional analytical tests were performed on the residue: IR spectral analysis, gas chromatography (GC), and high-performance liquid chromatography (HPLC). A gas chromatograph of the dissolved residue performed on a 30-m SE-30 (100\% methylsilicone) GC column showed no detectable peaks beyond those of the solvent. This would 


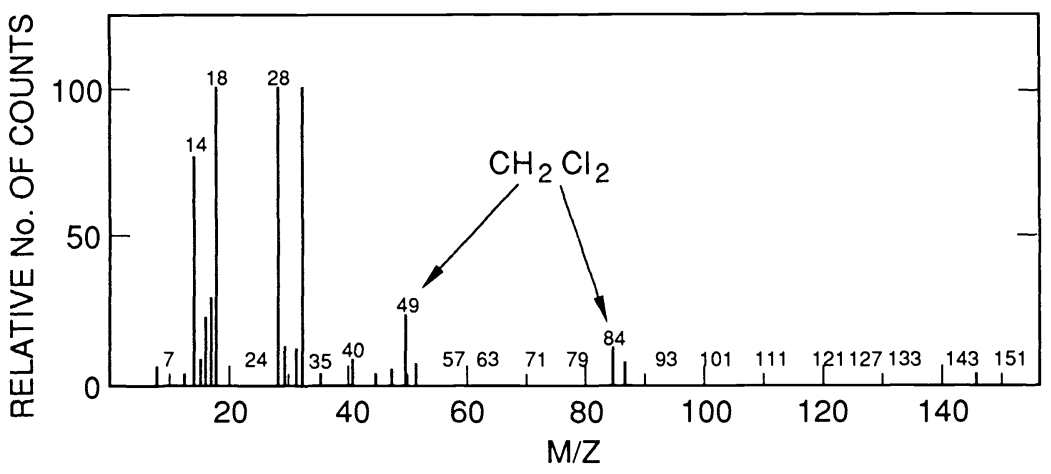

Fig. 2 Mass spectrum of Spectralon sample outgassed under vacuum $\left(10^{-6}\right.$ Torr) for $5 \mathrm{~min}$ at $22^{\circ} \mathrm{C}$.

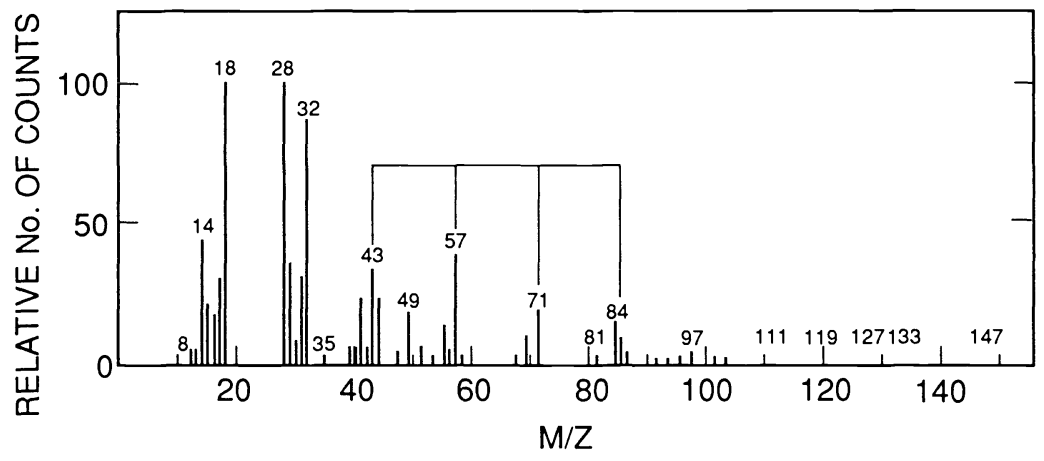

Fig. 3 Mass spectrum of Spectralon sample outgassed under vacuum $\left(10^{-6}\right.$ Torr $)$ for $1.5 \mathrm{~h}$ at $95^{\circ} \mathrm{C}$.

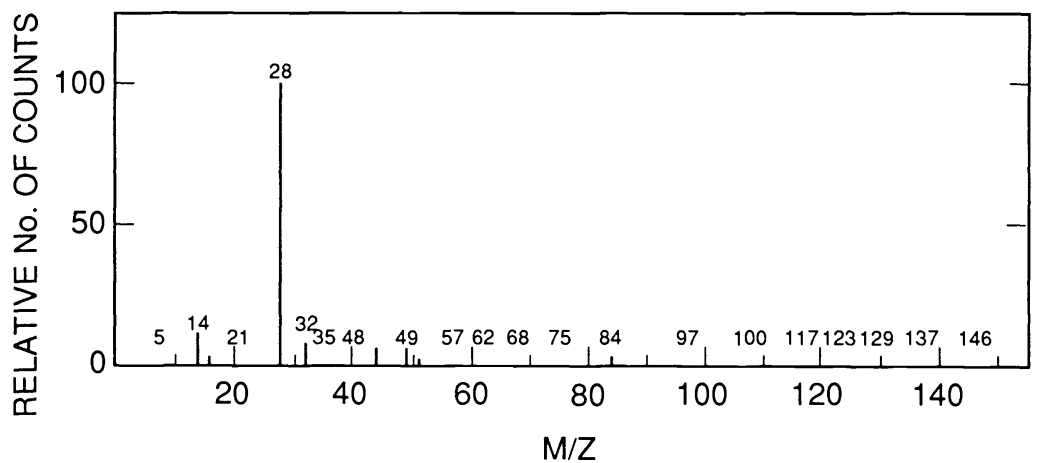

Fig. 4 Mass spectrum of Spectralon sample outgassed under vacuum $\left(10^{-6}\right.$ Torr) for $24 \mathrm{~h}$ at $90^{\circ} \mathrm{C}$.

be an expected result if the impurity had a highly polar functional group (acid, alcohol, etc.) that prevented its passage through the GC column. IR spectra of the residue (Fig. 5) confirms aspects of our analysis but, unfortunately, does not permit an unambiguous identification of the compound. The broadband at $3400 \mathrm{~cm}^{-1}$ corresponds to hydroxyl $(\mathrm{OH})$ groups possibly from absorbed water or from a carboxylic acid or an alcohol group, while the sharp peaks at $2900 \mathrm{~cm}^{-1}$ are the $\mathrm{C}-\mathrm{H}$ stretches of the hydrocarbon. The strong series of peaks at 1590,1475 , and $1150 \mathrm{~cm}^{-1}$ cannot be unambiguously assigned but may correspond to carboxylic acid, ester, or amide functional groups-all of which are highly polar. The inability to absolutely identify the compound with the IR spectrum is exacerbated by the possibility that there may be several components in the residue.

High-performance liquid chromatography (Fig. 6) was performed on the residue using two-column gel permeation chromatography (GPC) with tetrahydrofuran solvent. The detectors used were a differential refractometer and a UV detector set at $254 \mathrm{~nm}$. GPC works on the size exclusion principle and yields accurate molecular weights when calibrated. The GPC of the residue suggests that it is comprised, in fact, of several components of differing masses.

The GPC spectrum show multiple components with molecular weights ranging from approximately 200 to $560 \mathrm{amu}$, with an average of about $300 \mathrm{amu}$. While the analytical analysis performed on the impurities extracted from the 


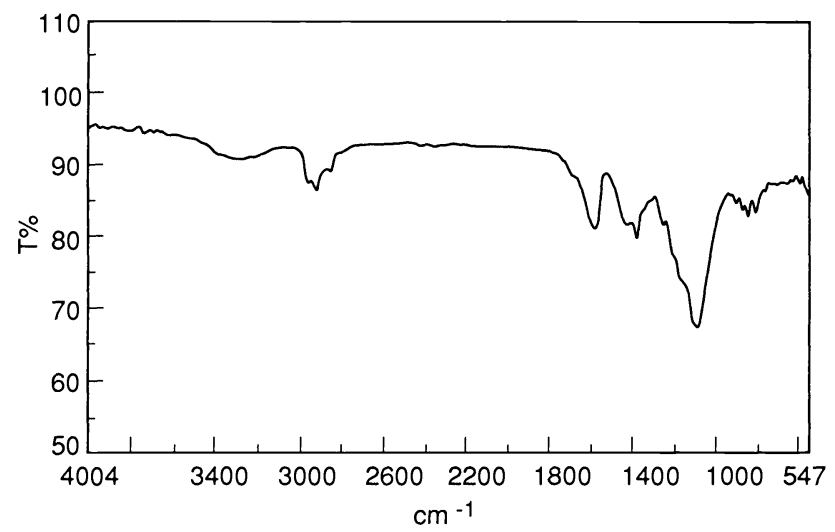

Fig. 5 IR spectrum of extracted impurities from Spectralon mixed with $\mathrm{KBr}$ and recorded as a diffuse reflectance spectrum.

Spectralon material did not permit an absolute identification of the compounds involved, several conclusions can be drawn. The impurity appears to be volatile and multicomponent, with a relatively high molecular weight range and some type of polar functional group: possibly long-chain aliphatic acids or esters. These impurities could be introduced from the surfactant used in preparation of the PTFE resin or from oil or lubricant used in the processing of the resin.

\subsection{UV Stability of Cleaned Spectralon}

Following the procedure previously defined we took a fresh Spectralon sample and pumped and baked it out at $90^{\circ} \mathrm{C}$ under $10^{-6}$ Torr vacuum for $24 \mathrm{~h}$. At the end of this period the sample was sealed off and allowed to outgas for $2 \mathrm{~h}$. Mass spectral analysis of the effluents showed that almost no hydrocarbon contaminant was being expelled. This clean sample was then exposed in the solar UV testing at TRW. The hemispheric reflectance properties of this cleaned material stabilized during exposure to vacuum UV. ${ }^{2}$ Also, the total solar absorptivity changed from 0.026 in the unexposed sample to 0.05 in the exposed sample. This supports the initial contention that the degradation is caused by a volatile contaminant and that the material can be UV stabilized by the relatively simple vacuum-bake-out procedure outlined above.

\subsection{Reabsorption of Volatile Impurities by Spectralon}

While this study has established that Spectralon is stable to UV radiation after the removal of the volatile impurities, to be determined is whether the material may have a tendency to reabsorb volatile organics from the air. Given the nonpolar nature of PTFE, it is entirely conceivable that organics, particularly nonpolar hydrocarbons, could be reabsorbed into the cleaned material. If significant reabsorption occurs, it may have an impact on the handling and deployment of the material in spacecraft applications. To test this, two Spectralon samples were vacuum baked at $10^{-7}$ Torr at a temperature of $90^{\circ} \mathrm{C}$ for 1 week, after which time no volatile impurities were detected by mass spectral analysis. The two samples were then exposed to several types of potential volatile impurities in an open environment for a period of 1 week. One sample was exposed to the aromatic hydro-

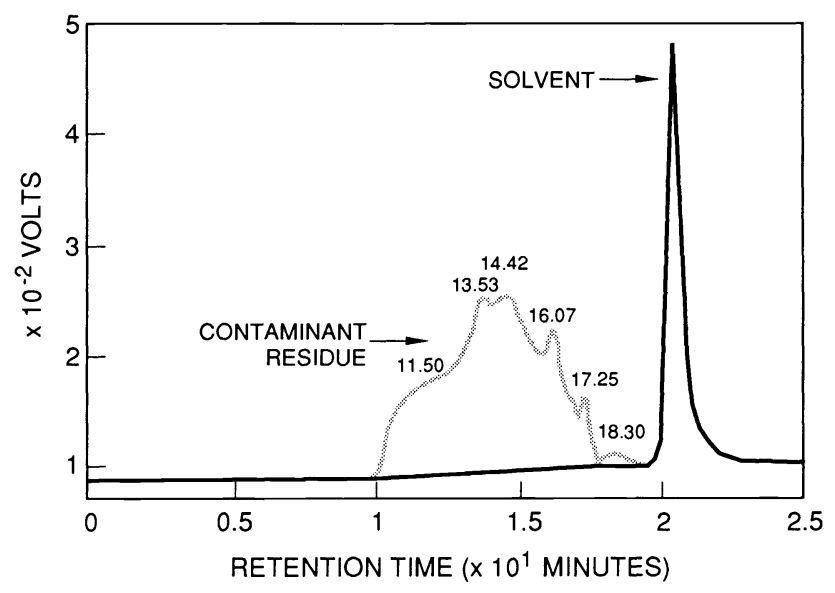

Fig. 6 High-performance liquid chromatograph of impurity residue extracted from Spectralon material.

carbons, toluene and napthalene, while the other was exposed to a saturated hydrocarbon, eicosane and silicone oil. These materials were selected as representative types of volatile materials that Spectralon would likely be exposed to in spacecraft applications. After the 1-week exposure, the samples were evacuated, the temperature raised to $90^{\circ} \mathrm{C}$, and the emitted volatiles measured by mass spectral analysis. The sample exposed to silicone oil and eicosane hydrocarbons showed no evidence of absorbed silicone oil and only a trace amount of the hydrocarbon. It is significant that silicone oil was not absorbed because it is a ubiquitous contaminant in spacecrafts. Similarly, it is interesting that a nonpolar medium such as PTFE fails to absorb simple hydrocarbons. This experiment suggests that reabsorption by the Spectralon of these two general classes of compounds will not pose a significant problem when handling previously cleaned material. The results of the reabsorption experiment for aromatic compounds are considerably different. The mass spectral analysis showed that significant amounts of both the toluene (mass 91) and napthalene (mass 128) were reabsorbed and outgassed even at room temperature. Whether this poses a problem for the handling and deployment of Spectralon depends on how common aromatics are in the spacecraft environment and whether or not the absorbed aromatics will affect UV stability. In general, it may be advisable to seal the Spectralon sample during integration and prior to launch to inhibit contaminant reabsorption. However, note that any contaminants absorbed from the atmosphere at ambient pressure must be relatively volatile (or they would not be in the atmosphere in significant quantities) and would, therefore, outgas from the Spectralon on entering the vacuum of space. Taken together, this data suggests that some types of potential contaminants can reabsorb into the material, however, overall, the reabsorption issue does not appear to be significant.

\section{Resin Study}

This section contains the results of an investigation of the volatile hydrocarbon impurity found in the PTFE resins used to make the final Spectralon product. A total of five different resin powders from different primary vendors were investigated to ascertain whether any volatile hydrocarbon im- 
purities were present in the resin prior to the manufacture of Spectralon and, if so, in what relative quantities.

\subsection{Experimental Methodology}

The resin samples evaluated $\mathrm{A}, \mathrm{B}, \mathrm{C}, \mathrm{D}$, and $\mathrm{E}$, were supplied by Labsphere from their stock of resins used to manufacture Spectralon. These resins were supplied by three different manufacturers: one manufacturer supplied $B$, another supplied C and E, while a third supplied A. Resin D is no longer available, but because it was the industry standard for years, it is included for comparison. For the test, a 4-g sample of the resin powder was weighed into a glass container fitted with a sintered glass cap. The container was then loaded into a sample chamber and attached to the manifold of the gas-sampling device on the mass spectrometer (Finnigan 3200). The sample chamber was mechanically evacuated for $30 \mathrm{~min}$ at room temperature followed by 1 min of ion pumping, at which point the sample was sealed off and allowed to outgas for $5 \mathrm{~min}$. The effluent was then let into the mass spectrometer and the spectrum was determined. This procedure was repeated with another $1 \mathrm{~min}$ of ion pumping and $5 \mathrm{~min}$ of outgassing, at which time the room temperature spectrum was again measured. Following the room temperature measurements, the sample was ion pumped for $5 \mathrm{~min}$, sealed off, brought to $50^{\circ} \mathrm{C}$, and allowed to outgas for $1 \mathrm{~h}$, after which time the effluent was let into the mass spectrometer for analysis. Finally this procedure was repeated with the sample being ion pumped for $5 \mathrm{~min}$ at $50^{\circ} \mathrm{C}$, sealed off and taken to $90^{\circ} \mathrm{C}$, where it outgassed for $1 \mathrm{~h}$, at which point the mass spectrum of the effluents was determined.

\subsection{Experimental Results}

All the samples showed similar mass spectra that were characterized by a large peak at mass 18 resulting from residual water and at mass 28 , which can be attributed to a number of species such as $\mathrm{N}_{2}, \mathrm{CO}$, or $\mathrm{C}_{2} \mathrm{H}_{4}$. More importantly, all of the resins showed the presence of an organic effluent by the characteristic hydrocarbon fragmentation pattern occurring at masses $43\left(\mathrm{C}_{3} \mathrm{H}_{7}\right), 57\left(\mathrm{C}_{4} \mathrm{H}_{9}\right), 71\left(\mathrm{C}_{5} \mathrm{H}_{11}\right)$, and 85 $\left(\mathrm{C}_{6} \mathrm{H}_{13}\right)$. A representative mass spectrum for the $\mathrm{E}$ resin taken after the $90^{\circ} \mathrm{C}$ outgassing is shown in Fig. 7. A complete set of mass spectral data for all of the resins at room temperature, 50 , and $90^{\circ} \mathrm{C}$ were obtained. While the mass spectral analysis confirms the presence of an organic contaminant, it does not permit its exact chemical identification. Furthermore, it is also possible that more than one organic impurity contributing to the observed spectrum exists. As mentioned previously, all of the samples investigated showed the presence of an organic impurity that was detectable at room temperature in some of the resins and readily detectable at $90^{\circ} \mathrm{C}$ in all of the samples. Note that the fragmentation pattern for all of the samples is extremely similar, suggesting that there may be a common impurity. The spectra were also identical to those obtained from the effluent outgassed from the solid Spectralon reflectance samples. The data obtained from the mass spectral analysis does allow the relative amounts of organic contaminant present in the resins to be determined. The relative quantities are determined from the mass chromatograms by comparing the average number of counts obtained from the two most intense hydrocarbon mass peaks (57 and 71) evolved at $90^{\circ} \mathrm{C}$. Be-

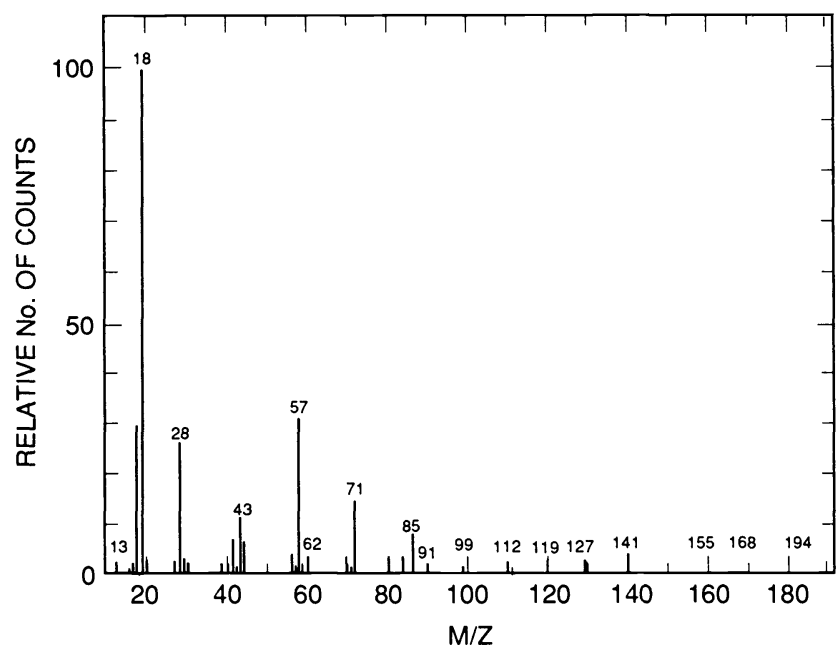

Fig. 7 Mass spectrum of organic effluents emitted from resin $E$ at $90^{\circ} \mathrm{C}$.

Table 2 Relative quantities of volatile hydrocarbon-based impurities emitted from PTFE resins.

\begin{tabular}{|l|l|l|}
\hline & \multicolumn{2}{|l|}{$\begin{array}{l}\text { Number of } \\
\text { Counts }\end{array}$} \\
\hline Resin & \multicolumn{1}{|c|}{ Mass 57 } & \multicolumn{1}{c|}{ Mass 71 } \\
\hline \hline A & 4168 & 2216 \\
\hline B & 8024 & 5728 \\
\hline C & 26208 & 14192 \\
\hline D & 34368 & 23872 \\
\hline E & 97408 & 48704 \\
\hline
\end{tabular}

cause the amount of contaminant emitted is proportional to the number of counts, the resins can be ordered by increasing amounts of contamination. This data is presented in Table 2 in which the average number of counts for mass 57 and 71 for each resin are listed. In general, resin A was the cleanest, followed by B, C, D, with E being the most contaminated.

Clearly there is a significant range in the relative amounts of volatile contaminants contained in the resins. The most contaminated resin, E, contains approximately 20 times more effluent than the cleanest resin, A. While none of the resins were absolutely free from volatile organic impurities, resin A and B were substantially better than the other three, and would be expected to produce Spectralon that required a minimal amount of decontamination. Note that the experiments reported here analyze only the volatile organic contained in the resins and not any nonvolatiles that may be present. However, because our preliminary work has clearly demonstrated the relationship between UV stability and organic volatiles, the use of Spectralon material made from resin A or B should be inherently more stable.

\section{Spectralon Handling Procedure}

In summary, the Spectralon reflectance material, as received from the manufacturer, contains some volatile hydrocarbon impurities. These impurities are contained in the PTFE resin 
from which Spectralon is manufactured, but can be easily removed by a vacuum bake-out procedure. A significant point is that, except for this volatile impurity, Spectralon is an otherwise clean material that will only become more contaminated by washing in water or, worse, organic solvents.

Spectralon should always be handled with white cotton lint-free gloves in a clean environment (preferably a clean room). More specifically, it should not be exposed to volatile organics after the cleaning procedure. By vacuum-baking Spectralon $\left(10^{-6}\right.$ Torr at $\left.90^{\circ} \mathrm{C}\right)$ for a period of $48 \mathrm{~h}$, all of the volatile organics are readily removed. This is the only cleaning procedure that we recommend. As mentioned previously, washing with water or solvents will only contaminate the material further.

After the vacuum bake-out procedure, the Spectralon should again be handled with cotton gloves in a clean environment. Because the material will have a tendency to reabsorb volatile organics, it should be stored and transported only in clean air-tight containers, preferably glass or oil-free metal containers. Under no circumstances should it be placed in nonspace qualified plastic containers or bags, because plasticizers and organic antistatic agents can readily leach from those containers and recontaminate the material. If plastic containers must be used, we recommend Fluo- roware ${ }^{\text {(TM }} \mathrm{H} 22$ Series individual wafer shipping trays (Fluoroware Inc., Chaska, Minnesota) (used to ship semiconductor wafers), or $3 \mathrm{M}$ brand $2110 \mathrm{E}$ embossed recloseable static-shielding bags. The material should be left in the sealed container until integrated into the spacecraft.

\section{Acknowledgments}

TRW UV exposure and hemispheric reflectance data were acquired by M. R. Gilmore. Chemical analysis testing was conducted by G. Plett of JPL. The research described in this paper was carried out by JPL, California Institute of Technology, under contract with the National Aeronautics and Space Administration.

\section{References}

1. M. R. Gilmore, "Ultraviolet degradations of Spectralon samples for the Jet Propulsion Laboratory," TRW Space Technology Group Internal Document L123.16g-90-16 (March 1990).

2. C. J. Bruegge, A. E. Stiegman, R. A. Rainen, and A. W. Springsteen, "Use of Spectralon as a diffuse reflectance standard for in-flight calibration of earth-orbiting sensors," Opt. Eng. 32(4), in this issue (1993).

Biographies and photographs of the authors appear with the paper "Use of Spectralon as a diffuse reflectance standard for in-flight calibration of earth-orbiting sensors" in this issue. 